\title{
Sodium-glucose cotransporter-2 inhibitors: updated evidence on their efficacy and safety in patients with type-2 diabetes
}

\author{
Vipul Gupta $^{1 *}$, Girish Khurana ${ }^{2}$
}

\author{
${ }^{1}$ Gupta Ultrasound and Heart Care Center, Paschim Vihar, New Delhi, India \\ ${ }^{2}$ Vidya Medicare Center, Bahadur Garh, Haryana, India
}

Received: 24 February 2020

Revised: 14 March 2020

Accepted: 16 March 2020

\section{*Correspondence:}

Dr. Vipul Gupta,

Email: guptasvipul@hotmail.com

Copyright: (c) the author(s), publisher and licensee Medip Academy. This is an open-access article distributed under the terms of the Creative Commons Attribution Non-Commercial License, which permits unrestricted non-commercial use, distribution, and reproduction in any medium, provided the original work is properly cited.

\begin{abstract}
Management of type-2 diabetes mellitus (T2DM) is challenging. The scope of existing therapies toward T2DM has transformed remarkably. These large assortments of therapies have produced evidence-based data. Sodium-glucose cotransporter-2 inhibitor (SGLT-2i) is the most recent class of oral anti-hyperglycemic agents. They are approved by Food and Drug Administration for the treatment of diabetes mellitus. SGLT-2i has a unique mechanism of action and that lower glucose independent of insulin. They reduce renal tubular glucose reabsorption, thereby lowering blood glucose without stimulating the release of insulin. Additional advantages involve suitable effects on blood pressure and weight. According to guidelines of the American Association of Clinical Endocrinologists/ the American College of Endocrinology 2016, SGLT-2i (in the form of canagliflozin, dapagliflozin, and empagliflozin) is one of the acceptable alternatives to metformin as initial therapy towards T2DM. Canagliflozin, dapagliflozin, and empagliflozin reduce the cardiovascular risk in comparison to placebo as the part of standard care. This review article focuses on the clinical trials published over the past year and specifically the metabolic aspect of SGLT-2i and the adverse effects related to SGLT-2 inhibitors.
\end{abstract}

Keywords: SGLT2, Type 2 diabetes mellitus, Oral glucose-lowering medicines

\section{INTRODUCTION}

The estimated total prevalence of type-2 diabetes in India in 2017 was $8.8 \%$, with approximately 7.2 million cases. ${ }^{1}$ Type-2 diabetes mellitus (T2DM) is a chronic progressive disease characterized by hyperglycemia that results from insulin resistance, diminished or absent insulin secretion, or both. Morbidity and mortality associated with diabetes are high, resulting from a spectrum of complications, primarily cardiovascular disease and nephropathy. ${ }^{2}$

Glucose-lowering therapies with insulin-dependent mechanisms of action loose efficacy over time as both the endogenous insulin secretion and insulin sensitivity decrease. Critical issues encountered in the management of diabetes are hypoglycemia and weight gain. These issues are pertinent specifically to the cardio vascular disease afflicted and obese patients, who comprise the largest subgroup population suffering from diabetes. ${ }^{3}$ Sodium-glucose cotransporter-2 (SGLT-2) inhibitors are the most recent class of anti-hyperglycemic agents to be awarded with the Food and Drug Administration (FDA) approval. SGLT-2 inhibitors function through a novel mechanism of reducing renal tubular glucose reabsorption, producing a reduction in blood glucose without stimulating insulin release. ${ }^{4}$ Other benefits may include favorable effects on blood pressure and weight. This benefit, coupled with significant glycemic and non- 
glycemic effects that include modest weight loss and an incidence of hypoglycemia similar to metformin, dipeptidyl peptidase-4 inhibitors (DPP-4i), glucagon-like peptide-1 receptor agonists (GLP-1 RA), and thiazolidinediones makes the SGLT-2 class of medications an essential option for type-2 diabetes mellitus (T2DM) as an alternative to metformin or as part of dual and triple therapy. The scope of this review will focus on clinical trials published over the past year and especially the metabolic impact of this class of medication, the remarkable results of clinical safety trials, and the adverse effects associated with these SGLT-2 inhibitors.

\section{MODE OF ACTION AND CLINICAL PHARMACOLOGY}

SGLT-2 inhibitors work by inhibiting SGLT-2 in the proximal convoluted tubule (PCT). SGLT-2 also prevents the reabsorption of glucose. This causes the excertion of glucose, which in turn causes improvement in most of the glycemic parameters. ${ }^{4}$

Dapagliflozin is approved as a $10 \mathrm{mg}$ once-daily drug, as monotherapy, or in combination with dipeptidyl peptidase-4 (DPP-4) inhibitors, sulfonylurea (SU), metformin, and/or insulin. Upon oral administration, this drug is promptly metabolized attaining the peak concentration in plasma within 2 hours. Dapagliflozin exhibit good measure (around 78\%) of oral bioavailability. The enzyme uridine diphosphateglucuronosyltransferase (UGT) plays key role in the hepatic and renal metabolism of dapagliflozin. This drug has been approved for prescribtion alone for use by diabetic patients who cannot safely consume metformin or have intolerance towards metformin. ${ }^{5}$

The oral bioavailiblity for canagliflozin is around $65 \%$. The peak plasma concentration of canagliflozin is achieved between 1 to 2 hours upon its oral administration. Whereas, canagliflozin takes around four to five days to achieve steady state. Two enzymes i.e. UGT1A9 and UGT2B4 perform key role in the metabolism of canagliflozin. Both the enzymes undertake the process of glucuronidation for metabolism of canagliflozin. The dosage regimen of canagliflozin begins with $100 \mathrm{mg}$ daily before the first meal of the day. In few diabetic patients showing inadequate response against the initial dose, the dosage of canagliflozin can be increased (up to $300 \mathrm{mg}$ ). In such cases, it is essential to maintain their renal parameters within the normal limits. ${ }^{6}$

Empagliflozin take six days to achieve the steady state. However, the peak plasma concentrations can be achieved between 1.33 to 3 hours upon its oral administration. ${ }^{7}$ Study by Hershon reported dose-dependent glycated hemoglobin reduction, upon the empagliflozin monotherapy regimen. This drug can be administered in once-daily frequency and with no relation to the meal timings. ${ }^{8}$

\section{GLYCEMIC EFFECTS OF SGLT-2 INHIBITORS IN T2DM SUBJECTS}

The glucose-lowering effect of SGLT-2 inhibitors is modest but comparable to other classes of oral antidiabetic medications. In placebo/active-controlled randomized trials, SGLT-2 inhibitors induced a mean reduction in glycated hemoglobin (HbA1c) of $\sim 0.7 \%$ (ranging from $0.4 \%$ to $1.1 \%$, depending on the baseline HbA1c). In these studies, the SGLT-2 inhibitor was used either as monotherapy or in combination with metformin, sulfonylureas, pioglitazone, sitagliptin, and/or insulin. In a meta-analysis of randomized controlled trials (RCTs) comparing SGLT-2 inhibitors with placebo (45 studies, $\mathrm{n}=11,232$ ), SGLT-2 induced a mean reduction of $0.66 \%$ in $\mathrm{HbA1c}$ [95\% confidence interval (CI) $-0.73 \%$ to $0.58 \%]$; this effect was similar in magnitude across the individual studies, where diverse background therapies were used. In addition, when compared with active comparators (including metformin, sulfonylurea, and sitagliptin) as either monotherapy or add-on treatment (13 studies, $n=5,175)$, SGLT-2 inhibitors also had a favorable effect in lowering HbA1c [-0.06\% (95\% CI-0.18\% to $0.05 \%)$ ]. ${ }^{9}$ A meta-analysis of 12 RCTs comparing SGLT2 inhibitors with incretins showed significant reductions in HbA1c (SMD -0.22; 95\% CI:-0.30, -0.14; p=0.000) and fasting plasma glucose (SMD-0.48; 95\% CI: -0.56 , $0.41 ; \mathrm{p}=0.000)$ with SGLT-2 inhibitors as compared to DPP-4 inhibitors. ${ }^{10}$ In addition, these reductions increased with a prolonged treatment duration from 12 to 78 weeks. A study by Cherukuri et al showed approximately 6 to 8 years duration of effect for the SGLT-2 inhibitors before the A1c returns to baseline and, it appears to be a better choice over other antidiabetic medications. ${ }^{11}$ In a 24 -week parallel-group, double-blind, placebo-controlled trial with patients on metformin $(n=546)$, dapagliflozin significantly improved glycemic control with mean HbA1c changes of $-0.3 \%$ with placebo, $-0.67 \%$ with $5 \mathrm{mg},-0.7 \%$ with 10 mg. ${ }^{12}$ In another 26-week randomized, double-blind, fourarm, parallel-group study in which patients on metformin were given placebo, sitagliptin $100 \mathrm{mg}$, or canagliflozin $100 \mathrm{mg}$ or $300 \mathrm{mg}$, those given canagliflozin (100 and $300 \mathrm{mg}$ ) had reduced HbA1c vs. placebo $(-0.79,-0.94$, and $-0.17 \%$, respectively). In a follow-up at week 52 , patients are given canagliflozin $100 \mathrm{mg}$, and $300 \mathrm{mg}$ demonstrated non-inferiority, and canaglifloz in $300 \mathrm{mg}$ demonstrated superiority to sitagliptin in lowering HbAlc $\left(-0.73,-0.88\right.$, and $-0.73 \%$, respectively). ${ }^{13}$ Canagliflozin provides glycaemic control, bodyweight reduction, and good tolerability in Indian patients with T2DM similar to that seen in patient populations across the world. Canagliflozin acts as an effective therapeutic option for patients with T2DM in India who may be on a range of background therapies.

A meta-analysis involving five trials and 4300 participants reported no significant reduction in changes in HbA1c with SGLT-2 inhibitors as compared with SUs (mean difference [MD] - 0.06; 95\% confidence interval [CI] [- 0.12, 0.08]). ${ }^{14}$ Incidentally, the recent study (2019) undertaken by Chen et al, reported reduced incidence of 
hypoglycemia in its study population as a consequence of using SGLT-2 inhibitors as an add-on to metformin. This observation was supported by statistical inputs in the form of odds ratio [OR] $0.12 ; 95 \% \mathrm{CI}[0.07,0.21]$. Further, this study concluded net weight reduction of around $3.5 \mathrm{~kg}$ in its study population as a consequence of using SGLT-2 inhibitors as an add-on to metformin. ${ }^{15}$

Regarding the anti-hyperglycemic potency of specific SGLT-2 inhibitors, there is a lack of head-to-head RCTs comparing the available SGLT-2 inhibitors. Indirect estimates have been obtained from network metaanalyses. Network analyses comparing canagliflozin, dapagliflozin, and empagliflozin found a tendency toward greater glucose-lowering efficacy of higher doses of canagliflozin over dapagliflozin and empagliflozin, respectively. Specifically, canagliflozin $300 \mathrm{mg}$ reduced HbA1c more than other SGLT-2 inhibitors, with the mean difference ranging from $0.20 \%$ to $0.64 \%$. However, the significance of these small differences between the SGLT-2 inhibitors is likely not clinically relevant. In diabetic patients with inadequately controlled glucose regulation despite high-dose insulin therapy, dapagliflozin may be an alternative combination choice to decrease the need for insulin dose and obtain an optimal $\mathrm{HbA1c}$, fasting plasma glucose levels and weight without significant side effects.

\section{EFFECTS OF SGLT INHIBITORS ON BODY WEIGHT}

Obesity and particularly visceral or abdominal obesity is associated with diabetes, insulin resistance, metabolic syndrome, and increased cardiovascular risk. Weight gain is a side effect of insulin therapy, sulfonylureas, and thiazolidinediones, whereas metformin and DPP-4 inhibitors are weight neutral. While, the GLP-1 agonists (most commonly the liraglutide and exenatide) are related with weight loss. Post-hoc pooled analysis of 10 RCTs involving the use of dapagliflozin in patients with T2DM $(n=5,163)$ reported a significant weight loss across all baseline BMI categories (including those with BMI of 18.5 to $<25 \mathrm{~kg} / \mathrm{m}^{2}$ by Lee et al. ${ }^{16}$ A recent meta-analysis by Wang et al. showed greater reductions in body weight (SMD -0.72; 95\% CI: $-0.81,-0.63 ; \mathrm{p}=0.000$ ) after the use of SGLT-2 inhibitors as compared to DPP-4 inhibitors. ${ }^{12}$ According to the results of the randomized controlled trial (2018) undertaken by Perna et al, the cognitive status of their study population remained same after the combination therapy of SGLT-2 inhibitors and incretins for a period of 1 year. This therapy resulted in elevated levels of high-density lipoprotein cholesterol (+5.73 $\mathrm{mg} / \mathrm{dl} ; \mathrm{p}<0.01)$. Additionally, this combination therapy caused net reduction in both the body weight (close to 2 $\mathrm{kg} ; \mathrm{p}<0.05)$ and BMI $\left(-0.69 \mathrm{~kg} / \mathrm{m}^{2} ; \mathrm{p}<0.05\right) .{ }^{17}$

\section{CARDIOVASCULAR DISEASE SAFETY}

The impact of glucose-lowering therapy on cardiovascular (CV) disease in T2DM has been controversial, with some reports yielding conflicting and even paradoxical findings. In response to this ongoing discussion, in 2008, the US FDA issued guidance for industry that mandated the demonstration of $\mathrm{CV}$ safety in the licensing of any new glucose-lowering drugs. The FDA required that, for a new medication for the treatment of T2DM, clinical trials in the drug development program must rule out unacceptable $\mathrm{CV}$ risk for a composite endpoint of major adverse $\mathrm{CV}$ events (MACE), comprising (at minimum) CV death, non-fatal myocardial infarction, and non-fatal stroke.

This FDA guidance has launched a new era of dedicated $\mathrm{CV}$ trials in T2DM, which are of great interest and clinical relevance as $\mathrm{CV}$ disease is the leading cause of death in individuals with T2DM. The SGLT-2 empagliflozin and canagliflozin have now been shown to reduce cardiovascular morbidity and mortality in patients with T2DM and a high cardiovascular risk, according to two landmark RCTs (EMPA-REG outcome and canvas); furthermore, there are beneficial renal effects with a reduction of albuminuria. The ongoing DECLARE-TIMI 58 and VERTIS cardiovascular RCTs, due for completion in 2019, are exploring the possible cardiovascular benefits and safety of dapagliflozin and ertugliflozin, respectively.

According to a network meta-analysis of 73 randomized clinical trials reporting cardiovascular risk with oral antihyperglycemic drugs in 101,183 patients, the relative risks of all-cause and cardiovascular mortality with SGLT-2 inhibitors were lower than with placebo, metformin, thiazolidinediones, or DPP- $4 \mathrm{i}^{18}$

In 2019, a systematic review and network meta-analysis by Grenet involved around 175,966 patients and 34 trials (from 1970 to 2018). This review compared the effects of old and new hypoglycemic drugs in T2D, on major adverse cardiovascular events (MACE) and mortality. Out of these 34 trials, fifty percent trails included major population of patients with previous cardiovascular history. This recent systematic review summarized that in comparison to the study controls, the SGLT-2 inhibitors were related with a reduced risk of overall mortality. The statistical representation is done by $\mathrm{OR}=0.84[95 \% \mathrm{CrI}$ : $0.74 ; 0.95]$ ). And, the combination of SGLT-2 inhibitors with GLP-1 agonists was associated with a reduced risk of major adverse cardiac events. This observation was supported by statistical inputs in the form of odds ratio (OR=0.89 [95\% CrI: 0.81;0.98] and OR=0.88 [95\% CrI: $0.81 ; 0.95]$, respectively). Further, in comparison to the DPP-4 inhibitors, SGLT-2 inhibitors showed reduced risk of overall mortality. The statistical representation is done by $\mathrm{OR}=0.82$ [95\% CrI: $0.69 ; 0.98] .{ }^{19}$

A network meta-analysis of 171,253 participants from 91 randomized controlled trials assessing the comparative effect of these anti-diabetic agents on heart failure outcomes reported that, SGLT-2 were significantly superior to insulin (OR: 0.75 , 95\% CI 0.62-0.91), dipeptidyl peptidase 4 inhibitors (OR: $0.68,95 \%$ CI 0.59 0.78 ), GLP-1 RA (OR: 0.65 , 95\% CI 0.54-0.78), and thiazolidinediones (OR: $0.46,95 \%$ CI $0.27-0.77$ ) in terms 
of heart failure risk. Furthermore, in an exploratory analysis among subjects with underlying heart failure or at risk of heart failure, the superiority of SGLT-2 was still significant. ${ }^{20}$

\section{EFFECTS OF SGLT INHIBITORS ON BLOOD PRESSURE}

Anti-hypertensive therapies are useful for majority of patients suffering from T2DM. Anti-hypertensive therapies regulate their blood pressure within the normal limits $(<140 / 90 \mathrm{mmHg})$.

The increased urinary glucose excretion caused by SGLT2 inhibitors cause osmotic diuresis and increased urinary frequency. This results in clinically significant blood pressure lowering, with a systolic BP reduction of $\sim 2.5$ $\mathrm{mm} \mathrm{Hg}$ and diastolic BP reduction of $\sim 1.5 \mathrm{~mm} \mathrm{Hg}$. Similarly, the study composing of 12 placebo studies reveal that the dapagliflozin treatment (10 $\mathrm{mg}$ daily) reduced diastolic blood pressure by $0.5 \mathrm{mmHg}$ and systolic blood pressure by $4.4 \mathrm{mmHg}$ as compared to the placebo group at $24^{\text {th }}$ week study period. ${ }^{21}$

A recent meta-analysis compared SGLT-2 inhibitors and DPP-4 inhibitors as second-line drugs in patients with type 2 diabetes found significant reduction in SBP (SMD -0.30 ; $95 \%$ CI: $-0.35,-0.25$; $=0.000$ ), and DBP (SMD 0.26 ; $95 \%$ CI: $-0.33,-0.20 ; \mathrm{p}=0.000)$ with SGLT-2i. ${ }^{12}$ The BP-lowering effect is unique for this anti-diabetic medication and possibly contributes to its observed cardio protective effect. Interestingly, clinical studies demonstrated that SGLT-2 inhibition is not associated with an increase in heart rate, despite this consistent drop in BP (tentolouris, 2019). This may represent an inhibitory effect on the usual baroreflex-mediated increase in sympathetic tone that accompanies a decrease in $\mathrm{BP}^{22}$ In 2019, a meta-analysis by Piperidou reported that SGLT-2 inhibitors reduced systolic and diastolic blood pressure by $4.43 \mathrm{mmHg}(95 \% \mathrm{CI}-5.24$ to -3.63$)$ and $1.81 \mathrm{mmHg}$ (95\% CI -2.38 to -1.23$)$, respectively. ${ }^{23}$

The factors of the empagliflozin-stimulated improvement of vascular parameters of arterial stiffness include variation in both systolic 24-h ambulatory blood pressure and high-sensitivity C-reactive protein, and, gender and age of the patient. On the other hand, factors measuring the variation in glucose metabolism and its volume revealed meager impact. This meta-analysis shows that SGLT-2 inhibitors provoke an average reduction of systolic/diastolic BP 3.62/1.70 mmHg in 24-h ambulatory BP. This BP-lowering effect remains unmodified regardless of the dose of SGLT-2 inhibitor and is comparable with BP-lowering efficacy of low-dose hydrochlorothiazide. ${ }^{24}$

\section{EFFECTS ON LIPID LEVELS}

The treatments with SGLT2 inhibitors decrease triglyceride levels and increase high-density lipoprotein
(HDL)-cholesterol levels. The meta-analysis published by Storgaard et al investigated the influence of several SGLT-2 inhibitors on serum lipids. ${ }^{25}$ The results of this investigation revealed an increment of levels of highdensity lipoprotein-C $(n=4,698$, mean difference 1.93 $\mathrm{mg} / \mathrm{dl})$ and low-density lipoprotein- $\mathrm{C}(\mathrm{n}=5,431$, mean difference $3.5 \mathrm{mg} / \mathrm{dl}$ ) in comparison to the placebo. This study also reported a depreciation in the levels of triglyceride levels $(\mathrm{n}=4,704$, mean difference $7.8 \mathrm{mg} / \mathrm{dl})$ in patients treated with SGLT-2 inhibitors. Canagliflozin was associated with the largest effects on serum lipids. Finally, in the same analysis, SGLT-2 inhibitors increased both HDL-C and LDL-C compared with other oral antidiabetic drugs (such as sulfonylureas and DPP4 inhibitors), although triglycerides were not significantly decreased. ${ }^{25}$ Another meta-analysis compared SGLT-2 inhibitors as an add-on to metformin monotherapy with other antidiabetics. In this meta-analysis, both LDL-C levels and HDL-C levels were elevated in the SGLT-2 inhibitor group by $2.5-9 \%$ and $7-9 \%$, respectively. ${ }^{26}$ Another systematic review reported significant elevation $(\mathrm{n}=18,684$, mean difference of $3.5 \mathrm{mg} / \mathrm{dl})$ in the levels of LDL-C. This phenomenon is explained by the hypothesis that the inhibition of SGLT-2 transfers energy sources to lipids from carbohydrates. This is apparent due to slight elevated levels of ketone after the treatment with SGLT-2 inhibitors. HDL-C levels were also increased significantly $(\mathrm{n}=18,684$, mean difference $3.9 \mathrm{mg} / \mathrm{dl})$. In the same review, treatment with canagliflozin revealed more promising results, whereas treatment with dapagliflozin could not alter levels of LDL-C or HDL-C. One possible reason for such result could be due to the rather small number of patients $(\mathrm{n}=808){ }^{27}$ The study undertaken by Hayashi et al reported the reduction of atherogenic small dense LDL particle levels upon intake of SGLT-2 inhibitors. This characteristic delivers supplementary long-term cardiovascular protection. Increased LDL cholesterol found with this therapy is because of reduced clearance of LDL from the circulation and greater lipolysis of triglyceride-rich lipoproteins. ${ }^{28}$ This study also reveal that when the patients were medicated with SGLT2-i, they showed low levels of BMI (-0.69 $\left.\mathrm{kg} / \mathrm{m}^{2} ; \mathrm{p}<0.05\right)$ and weight reduction $(-1.95 \mathrm{~kg} ; \mathrm{p}<0.05)$ but increased levels of high-density lipoprotein cholesterol (5.73 mg/dl; p<0.01).

\section{DECREASE IN ALBUMINURIA}

Historical studies have demonstrated that T2DM is related to a high risk of albuminuria due to an inflammatory response and oxidative stress induced by chronic hyperglycemia. Elevated albuminuria levels are associated with an incremental risk of microvascular complications (nephropathy, retinopathy, and neuropathy) and macrovascular events cardiovascular disease (CVD). Currently, increasing numbers of studies have shown a promising outcome with SGLT-2 inhibitors. Table 1 summarizes the results of SGLT-2 inhibitors on albuminuria. 
Table 1: Effects of SGLT2 inhibitors on albumin excretion rate.

\begin{tabular}{|l|lllllll|}
\hline Study & Subjects & $\begin{array}{l}\text { Investigation } \\
\text { drug }\end{array}$ & Comparator & Weeks & $\begin{array}{l}\text { Change in albumin excretion rate } \\
\text { Investigation } \\
\text { drug }\end{array}$ & Comparator & Difference \\
\hline Barnett et al $^{\mathbf{2 9}}$ & 375 & $\begin{array}{l}\text { Empagliflozin } \\
\text { 25 mg }\end{array}$ & Placebo & 52 & 155 & 79 & 184 \\
\hline Cefalu et a $^{\mathbf{3 0}}$ & 1450 & $\begin{array}{l}\text { Canagliflozin } \\
\text { 100/300 mg }\end{array}$ & Glimepiride & 52 & $0.1 / 0.9$ & 0.7 & $0.8 / 1.5$ \\
\hline Kohan et al $^{\mathbf{3 1}}$ & 252 & $\begin{array}{l}\text { Dapagliflozin } \\
5 / 10 \mathrm{mg}\end{array}$ & Placebo & 104 & $78.0 / 11.7$ & 69.7 & $8.3 / 81.4$ \\
\hline Yale et al $^{\mathbf{3 2}}$ & 269 & $\begin{array}{l}\text { Canagliflozin } \\
\text { 100/300 mg }\end{array}$ & Placebo & 52 & $117.5 / 96.2$ & 15.4 & $132.9 / 111.6$ \\
\hline
\end{tabular}

Along with the information presented in above table, the recent data also promote that SGLT-2 inhibitors cause reduction in albuminuria. Recently, Luo et al. performed a meta-analysis of $26 \mathrm{RCTs}$ involving 14,929 patients to evaluate the effects of novel antidiabetic drugs on albuminuria in patients with T2DM. Pooled analysis suggested that SGLT-2 inhibitors were associated with a reduction in albuminuria compared with other conventional therapies or placebo (WMD - 26.23\%, 95\% CI - 35.90 to $-16.56 ; \mathrm{p}<0.00001) .{ }^{33}$

\section{THE DECREASE IN URIC ACID LEVELS}

Evidence suggests that SGLT-2 inhibitors reduce serum uric acid levels by approximately $1 \mathrm{mg} / \mathrm{dl}$. Hyperuricemia is linked to insulin resistance, and some consider this to be a component of metabolic syndrome. Serum uric acid level, another component of insulin resistance or metabolic syndrome, is associated with an unfavorable $\mathrm{CV}$ endpoint. Several mechanisms have been postulated for the links between high uric acid levels and adverse CV outcomes. Hyperuricemia causes endothelial dysfunction with decreased nitric oxide levels at the macula densa. Chronic hyperuricemia causes vascular smooth muscle cell proliferation. It increases blood pressure through renal vasoconstriction by activation of the reninangiotensin system (RAS). The elevated uric acid level is associated with the development and progression of atherosclerosis through endothelial dysfunction and inflammation. Thus, a decrease in uric acid by SGLT-2 inhibitors might be a unique property of these drugs that contribute to reducing $\mathrm{CV}$ risk.

\section{APPLICATION OF SGLT-2 INHIBITORS IN T2DM PATIENTS SUFFERING FROM RENAL IMPAIRMENT}

In the year 2013, the drug canagliflozin received approval from FDA. However, post-approval FDA has received more than 100 confirmed cases of acute kidney injury with canagliflozin $(n=73)$ or dapagliflozin $(n=28)$. In 2016, the FDA warned about the risk of acute kidney injury for patients treated with SGLT-2 inhibitors including canagliflozin and dapagliflozin. (https://www. fda.gov/Drugs/DrugSafety/ucm505860.htm), though acute kidney injury was not increased with empagliflozin in the EMPA-REG outcome trial or with canagliflozin in the canvas program. However, empagliflozin can be used in patients with an estimated glomerular filtration rate (eGFR) $\geq 45 \mathrm{ml} / \mathrm{min} / 1.73 \mathrm{~m}^{2}$, whereas other SGLT-2 inhibitors are recommended for patients with an eGFR $\geq 60 \mathrm{ml} / \mathrm{min} / 1.73 \mathrm{~m}^{2}$. The permission to prescribe SGLT-2 inhibitors to patients with renal impairment varies according to an individual agent. Risk factors for acute kidney injury with SGLT-2 inhibitors include decreased blood volume, chronic renal impairment, heart failure, and the concomitant use of some medications (e.g., diuretics or RAS blockers). Furthermore, it is believed that the efficacy of SGLT-2 inhibitors is lower in subjects with a low eGFR because urinary glucose excretion in the presence of SGLT-2 inhibitors decreases with declining renal function. Of note, a recent study found that the effects of empagliflozin on clinical outcomes in patients with T2D, established CV disease, and chronic kidney disease were consistent across categories of eGFR and albuminuria. In a pooled analysis of 11 randomized controlled trials, dapagliflozin did not decrease HbA1c levels in patients with T2DM and an eGFR of 15$45 \mathrm{ml} / \mathrm{min} / 1.73 \mathrm{~m}^{2}$; however, it reduced blood pressure, albuminuria, and body weight significantly. Thus, despite the decrease in glucose-lowering efficacy with declining renal function, the beneficial effects of SGLT-2 inhibitors on clinical outcomes might be consistent in patients with renal impairment.

\section{ADVERSE EFFECTS AND SAFETY}

The most common adverse effect observed with SGLT-2 inhibitors is an increment in urogenital infections, which occurred in $4.5 \%$ more participants on empagliflozin than placebo in the EMPA-REG outcome trial and was also more frequent in participants taking canagliflozin as compared to placebo in the canvas program. The study carried out by Wang et al involved data from twelve placebo-controlled clinical trials involving dapagliflozin. ${ }^{10}$ The published data revealed the incidence of clinically diagnosed urogenital tract infection to around $4.1 \%, 5.7 \%$, and $4.8 \%$, when the dosage of dapagliflozin was maintained at $2.5,5$, and $10 \mathrm{mg}$ per day. Whereas, in the placebo group, the incidence of clinically diagnosed 
urogenital tract infection was recorded at $0.9 \%$. This meta-analysis show a higher incidence of genital infections (OR 4.49; 95\% CI: 2.96, 6.83; p=0.000) in diabetic patients receiving SGLT-2 inhibitors by Wang et al. ${ }^{10}$ Available literatures therefore recommend for not using SGLT-2 inhibitors towards the treatment of intractable or recurrent genital infection. ${ }^{12}$

It is known that SGLT-2 inhibitors follow non-insulinbased mechanism of action. Hence, the chances of hypoglycemia are nominal upon the usage of SGLT2i. However, when used in combination with metformin, SGLT-2 inhibitors may cause hypoglycemic conditions. A long-term (208-week) study by Nauck revealed drastic effects of SGLT2i on hypoglycemia. This study involved long-term comparison between glipizide plus metformin and dapagliflozin plus metformin, and further revealed ten-fold reduction in the prevalence of hypoglycemic episodes (5.4\% and 51.5\%, respectively). Additionally, in this study, the patients treated with glipizide plus metformin group encountered 1,191 hypoglycemic events, whereas, dapagliflozin plus metformin group encountered only 35 hypoglycemic events. ${ }^{34}$

The rate of bone formation is slightly affected by SGLT-2 inhibitors. SGLT-2 inhibitors usage has also been reported to raise the levels of bone resorption markers without affecting the bone mineral density. However, a long-term study (102-weeks) with dapagliflozin failed to establish any fluctuations in the levels of bone resorption markers, as compared to placebo when combined with metformin. ${ }^{4}$ Recently, a systematic literature review and Bayesian network meta-analysis involving $40 \mathrm{RCTs}$ and 32,343 T2DM patients showed 466 fracture cases with the use of SGLT-2 inhibitors. This review revealed negative correlation between SGLT-2 Inhibitors and the risk of fracture, as compared with the control group. This correlation is displayed as statistical inputs in the form of Odds ratio $=1.01,95 \% \mathrm{CI}$ in the range of $0.83-1.23$; $\mathrm{p}=0.91 ; \quad \mathrm{I} 2=27 \%$. This study further reported insignificant correlation between empagliflozin $(\mathrm{OR}=0.78,95 \%$ CI 0.23-2.80), canagliflozin $(\mathrm{OR}=0.57$, $95 \%$ CI $0.12-1.90)$, and dapagliflozin $(\mathrm{OR}=0.58,95 \% \mathrm{CI}$ $0.13-2.00)$, and the risk of fracture. ${ }^{35}$

A meta-analysis of 27 randomized controlled trials reported an increase in the incidence rate of peripheral artery disease with SGLT-2 inhibitors (MH-OR: 1.26 $[1.04,1.52])$. Although the increase of risk was statistically significant with canagliflozin, there is no reason to believe that empagliflozin or dapagliflozin increasing the risk of either peripheral artery disease of lower limb amputations. MH-OR for amputation in the three cardiovascular safety trials with SGLT-2 inhibitors was $1.22[0.59-2.52] .^{36}$

In 2019, a systematic review reported no precise association between interruption of SGLT2i and the occurrence of diabetic ketoacidosis. ${ }^{37}$ Recent study by Dicembrini report that the published data from the randomized trials used in their study do not suggest of harmful outcomes of SGLT-2. This study observed an upsurge in the prospect of UTI within the SGLT2 inhibitors groups in comparison to the study control groups, having placebo or other oral antidiabetic agents. ${ }^{36}$

\section{PLACE OF SGLT2 INHIBITORS IN THERAPY}

The Update to the position statement of the American Diabetes Association and the European Association for the Study of Diabetes recommends the use of SGLT-2 inhibitors as one of the second-line therapy when monotherapy with metformin fails. ${ }^{38}$ The AACE/ACE 2016 guidelines state that SGLT-2 inhibitors as one of the acceptable alternatives to metformin as initial therapy. ${ }^{39}$ The National Institute of Health and Care Excellence (NICE) has issued the recent guidance document on SGLT-2 inhibitors advocated empagliflozin, dapagliflozin or canagliflozin as a monotherapy alternative for managing T2DM in diabetic patients who cannot safely consume metformin or have intolerance towards metformin. $^{40}$

\section{CONCLUSION}

The SGLT-2 inhibitors class of medications possesses many glycemic and non-glycemic characteristics that make them an important option for individualizing therapy in patients with T2DM. SGLT-2 inhibitors is generally well-tolerated, with a low incidence of hypoglycemia. Adverse events related to urogenital infections, fractures, and acute kidney injuries are among the most common. A key benefit of canagliflozin, dapagliflozin, and empagliflozin is their ability to reduce $\mathrm{CV}$ risk compared to placebo as part of standard care. Thus, SGLT-2 inhibitors should be considered in that setting with the proper recognition of the associated side effects.

\section{Funding: No funding sources \\ Conflict of interest: None declared \\ Ethical approval: Not required}

\section{REFERENCES}

1. International Diabetes Federation. IDF Diabetic Atlas 7th Edition. http://www.idf.org/idf-diabetes-atlaseight-edition. Accessed 30 Aug 2017.

2. American Diabetes Association. Diagnosis and classification of diabetes mellitus. Diabetes care. 2013;36(Suppl 1):S67-74.

3. Lorenzati B, Zucco C, Miglietta S, Lamberti F, Bruno G. Oral hypoglycemic drugs: pathophysiological basis of their mechanism of actionoral hypoglycemic drugs: pathophysiological basis of their mechanism of action. Pharmaceuticals. 2010;3(9):3005-20.

4. Hsia DS, Grove O, Cefalu WT. An update on SGLT2 inhibitors for the treatment of diabetes mellitus. 
Current Opinion Endocrinol Diabetes Obesity. 2017;24(1):73.

5. van Baar MJ, van Ruiten $\mathrm{CC}$, Muskiet $\mathrm{MH}$, van Bloemendaal L, IJzerman RG, van Raalte $\mathrm{DH}$. SGLT2 inhibitors in combination therapy: from mechanisms to clinical considerations in type 2 diabetes management. Diabetes Care. 2018;41(8):1543-56.

6. Devineni D, Murphy J, Wang SS, Stieltjes H, Rothenberg P, Scheers E, et al. Absolute oral bioavailability and pharmacokinetics of canagliflozin: a microdose study in healthy participants. Clin Pharmacol Drug Develop. 2015;4(4):295-304.

7. Scheen AJ. Pharmacokinetic and pharmacodynamic profile of empagliflozin, a sodium glucose cotransporter 2 inhibitor. Clin Pharmacokinet. 2014;53(3):213-25.

8. Hershon KS. Options for empagliflozin in combination therapy in type 2 diabetes mellitus. Int $\mathbf{J}$ General Med. 2016;9:155.

9. Vasilakou D, Karagiannis T, Athanasiadou E, Mainou M, Liakos A, Bekiari E, et al. Sodiumglucose cotransporter 2 inhibitors for type 2 diabetes: a systematic review and meta-analysis. Annals Internal Med. 2013;159(4):262-74.

10. Wang Z, Sun J, Han R, Fan D, Dong X, Luan Z, et al. Efficacy and safety of sodium-glucose cotransporter2 inhibitors versus dipeptidyl peptidase- 4 inhibitors as monotherapy or add-on to metformin in patients with type 2 diabetes mellitus: A systematic review and meta-analysis. Diabetes Obesity Metabol. 2018;20(1):113-20.

11. Cherukuri L, Smith MS, Tayek JA. The durability of oral diabetic medications: Time to A1c baseline and a review of common oral medications used by the primary care provider. Endocrinol Diabetes Metabol J. 2018;2(3).

12. Filippatos TD, Liberopoulos EN, Elisaf MS. Dapagliflozin in patients with type 2 diabetes mellitus. Therap Advances Endocrinol Metabol. 2015;6(1):29-41.

13. Lavalle-González FJ, Januszewicz A, Davidson J, Tong C, Qiu R, Canovatchel W, et al. Efficacy and safety of canagliflozin compared with placebo and sitagliptin in patients with type 2 diabetes on background metformin monotherapy: a randomised trial. Diabetologia. 2013;56(12):2582-92.

14. Zaccardi F, Webb DR, Htike ZZ, Youssef D, Khunti $\mathrm{K}$, Davies MJ. Efficacy and safety of sodium-glucose co-transporter-2 inhibitors in type 2 diabetes mellitus: systematic review and network meta-analysis. Diabetes Obesity Metabol. 2016;18(8):783-94.

15. Chen Z, Li G. Sodium-Glucose Co-Transporter 2 Inhibitors Compared with Sulfonylureas in Patients with Type 2 Diabetes Inadequately Controlled on Metformin: A Meta-Analysis of Randomized Controlled Trials. Clin Drug Investigation. 2019;39(6):521-31
16. Lee PC, Ganguly S, Goh SY. Weight loss associated with sodium-glucose cotransporter-2 inhibition: a review of evidence and underlying mechanisms. Obesity Rev. 2018;19(12):1630-41.

17. Perna S, Mainardi M, Astrone P, Gozzer C, Biava A, Bacchio R, et al. 12-month effects of incretins versus SGLT2-Inhibitors on cognitive performance and metabolic profile. A randomized clinical trial in the elderly with Type-2 diabetes mellitus. Clin Pharmacol: Advances Applications. 2018;10:141.

18. Lee G, Oh SW, Hwang SS, Yoon JW, Kang S, Joh $\mathrm{HK}$, et al. Comparative effectiveness of oral antidiabetic drugs in preventing cardiovascular mortality and morbidity: A network meta-analysis. PloS one. 2017;12(5):e0177646.

19. Grenet G, Ribault S, Nguyen GB, Glais F, Metge A, Linet T, et al. Glucose Control Safety \& Efficacy in type 2 DIabetes, a systematic review and Network meta-analysis. PloS One. 2019;14(6):e0217701.

20. Yang DY, He X, Liang HW, Zhang SZ, Zhong XB, Luo $\mathrm{CF}$, et al. Comparative outcomes of heart failure among existent classes of anti-diabetic agents: a network meta-analysis of 171,253 participants from 91 randomized controlled trials. Cardiovascular Diabetol. 2019;18(1):47.

21. Reed JW. Impact of sodium-glucose cotransporter 2 inhibitors on blood pressure. Vascular Health Risk Management. 2016;12:393.

22. Tentolouris A, Vlachakis P, Tzeravini E, Eleftheriadou I, Tentolouris N. SGLT2 Inhibitors: A Review of Their Antidiabetic and Cardioprotective Effects. Int $\mathrm{J}$ Environ Res Public Health. 2019;16(16):2965.

23. Piperidou A, Sarafidis P, Boutou A, Thomopoulos C, Loutradis C, Alexandrou ME, et al. The effect of SGLT-2 inhibitors on albuminuria and proteinuria in diabetes mellitus: a systematic review and metaanalysis of randomized controlled trials. J Hypertension. 2019;37(7):1334-43.

24. Georgianos PI, Agarwal R. Ambulatory Blood Pressure Reduction With SGLT-2 Inhibitors: DoseResponse Meta-analysis and Comparative Evaluation With Low-Dose Hydrochlorothiazide. Diabetes Care. 2019;42(4):693-700.

25. Storgaard H, Gluud LL, Bennett C, Grøndahl MF, Christensen MB, Knop FK, et al. Benefits and harms of sodium-glucose co-transporter 2 inhibitors in patients with type 2 diabetes: a systematic review and meta-analysis. PLoS One. 2016;11(11):e0166125.

26. Simes BC, MacGregor GG. Sodium-Glucose Cotransporter-2 (SGLT2) Inhibitors: A Clinician's Guide. Diabetes, Metabolic Syndrome Obesity Targets Therapy. 2019;12:2125.

27. Cha SA, Park YM, Yun JS, Lim TS, Song KH, Yoo $\mathrm{KD}$, et al. A comparison of effects of DPP-4 inhibitor and SGLT2 inhibitor on lipid profile in patients with type 2 diabetes. Lipids Health Dis. 2017;16(1):58.

28. Hayashi T, Fukui T, Nakanishi N, Yamamoto S, Tomoyasu M, Osamura A, et al. Dapagliflozin decreases small dense low-density lipoprotein- 
cholesterol and increases high-density lipoprotein 2cholesterol in patients with type 2 diabetes: comparison with sitagliptin. Cardiovascular Diabetol. 2017;16(1):8.

29. Barnett AH, Mithal A, Manassie J, Jones R, Rattunde $\mathrm{H}$, Woerle $\mathrm{HJ}$, et al. Efficacy and safety of empagliflozin added to existing antidiabetes treatment in patients with type 2 diabetes and chronic kidney disease: a randomised, double-blind, placebocontrolled trial. Lancet Diabetes Endocrinol. 2014;2(5):369-84.

30. Cefalu WT, Leiter LA, Yoon KH, Arias P, Niskanen L, Xie J, et al. Efficacy and safety of canagliflozin versus glimepiride in patients with type 2 diabetes inadequately controlled with metformin (CANTATA-SU): 52 weeks results from a randomised, double-blind, phase 3 non-inferiority trial. The Lancet. 2013;382(9896):941-50.

31. Kohan DE, Fioretto P, Tang W, List JF. Long-term study of patients with type 2 diabetes and moderate renal impairment shows that dapagliflozin reduces weight and blood pressure but does not improve glycemic control. Kidney Int. 2014;85(4):962-71.

32. Yale JF, Bakris G, Cariou B, Nieto J, David-Neto E, Yue D, et al. Efficacy and safety of canagliflozin over 52 weeks in patients with type 2 diabetes mellitus and chronic kidney disease. Diabetes, Obesity Metabol. 2014;16(10):1016-27.

33. Luo Y, Lu K, Liu G, Wang J, Laurent I, Zhou X. The effects of novel antidiabetic drugs on albuminuria in type 2 diabetes mellitus: A systematic review and meta-analysis of randomized controlled trials. Clinical drug investigation. 2018;38(12):1089-108.

34. Nauck MA, Del Prato S, Meier JJ, Durán-García S, Rohwedder K, Elze M, et al. Dapagliflozin versus glipizide as add-on therapy in patients with type 2 diabetes who have inadequate glycemic control with metformin: a randomized, 52-week, double-blind, active-controlled noninferiority trial. Diabetes care. 2011;34(9):2015-22.
35. Azharuddin M, Adil M, Ghosh P, Sharma M. Sodium-glucose cotransporter 2 inhibitors and fracture risk in patients with type 2 diabetes mellitus: A systematic literature review and Bayesian network meta-analysis of randomized controlled trials. Diabetes Res Clin Pract. 2018;146:180-90.

36. Dicembrini I, Tomberli B, Nreu B, Baldereschi GI, Fanelli F, Mannucci E, et al. Peripheral artery disease and amputations with Sodium-Glucose coTransporter-2 (SGLT-2) inhibitors: A Meta-analysis Of Randomized Controlled Trials. Diabetes Res Clin Pract. 2019;153:138-44.

37. Thiruvenkatarajan V, Meyer EJ, Nanjappa N, Van Wijk RM, Jesudason D. Perioperative diabetic ketoacidosis associated with sodium-glucose cotransporter-2 inhibitors: a systematic review. $\mathrm{Br} \mathrm{J}$ Anaesthesia. 2019;123(1):27-36.

38. Shin JI. Second-line Glucose-Lowering Therapy in Type 2 Diabetes Mellitus. Current diabetes reports. 2019;19(8):54.

39. Garber A, Handelsman Y, Einhorn D, Bergman D, Bloomgarden Z, Fonseca V, et al. Diagnosis and management of prediabetes in the continuum of hyperglycemia - when do the risks of diabetes begin? A consensus statement from the American College of Endocrinology and the American Association of Clinical Endocrinologists. Endocrine Practi. 2008;14(7):933-46.

40. Johnston R, Uthman O, Cummins E, Clar C, Royle P, Colquitt $\mathrm{J}$, et al. Canagliflozin, dapagliflozin and empagliflozin monotherapy for treating type 2 diabetes: systematic review and economic evaluation. Health Technology Assessment. 2017;21(2):1-218.

Cite this article as: Gupta V, Khurana G. Sodiumglucose cotransporter-2 inhibitors: updated evidence on their efficacy and safety in patients with type-2 diabetes. Int J Basic Clin Pharmacol 2020;9:666-73. 